\title{
Mucoceles de cavidades paranasales. Experiencia en el Hospital Barros Luco-Trudeau
}

\author{
Paranasal sinus mucoceles. The Barros Luco-Trudeau experience
}

Jaime Osorio M¹, Daniel Muñoz S², Javiera Pardo J1, Maritza Rahal E1.

\begin{abstract}
RESUMEN
Introducción: Los mucoceles son pseudotumores expansivos de las cavidades paranasales, cuyos síntomas están dados por su crecimiento expansivo. El tratamiento tradicional ha sido la extracción quirúrgica por vía externa, siendo desplazado en las últimas décadas por la resección endoscópica.

Objetivo: Evaluar la experiencia en nuestro servicio sobre el diagnóstico y tratamiento de los mucoceles.

Material y método: Revisión retrospectiva de pacientes tratados por mucocele en el Servicio de Otorrinolaringología, entre 2002 y 2013. Se describen características clínicodemográficas, métodos diagnósticos, tratamiento y aparición de recidivas.

Resultados: Se analizaron 45 pacientes con seguimiento promedio de 24,93 meses. El 51,11\% correspondió al sexo masculino. La edad promedio fue 49,68 años. El 46,6\% fueron mucoceles fronto-etmoidales ( 6 frontales, 6 etmoidales y 9 fronto-etmoidales), el resto en seno maxilar, con un periodo de latencia de 7,33 meses. Los síntomas más frecuentes fueron oftalmológicos (55,5\%) como proptosis y diplopía, mientras que el $51,1 \%$ de los pacientes tenía antecedentes de rinosinusitis crónica. El tratamiento fue mediante resección endoscópica en el 66,22\%, por vía abierta 31,1\% y manejo combinado en $6,66 \%$. Hubo 8 casos recidivados, que representan el $17,7 \%$ de la serie.

Discusión: Nuestra casuística resultó semejante a otras series publicadas. En los últimos años se ha preferido el abordaje endoscópico, sin embargo, el abordaje externo o combinado siguen siendo claves en el manejo de mucoceles extensos o para tratar recidivas. La aparición potencialmente tardía de recidivas requiere un tiempo de seguimiento largo para estos pacientes.

Conclusiones: Los mucoceles representan un desafío diagnóstico y terapéutico y el manejo endoscópico parece una alternativa segura en el manejo de mucoceles que no comprometan órbita o tengan extensión craneal.
\end{abstract}

Palabras clave: Mucoceles, cavidades paranasales, cirugía endoscópica nasosinusal.

1 Médico. Servicio de Otorrinolaringología, Hospital Barros Luco-Trudeau.

2 Médico. Universidad de Chile. 


\section{ABSTRACT}

Introduction: Mucoceles are expansive pseudotumors of the paranasal sinuses and clinical presentations of symptoms are given by expansive growth. Traditional treatment has been open surgical extraction, being shifted in recent decades by endoscopic resection.

Aim: To evaluate our experience in diagnosis and treatment of mucoceles.

Material and methods: Retrospective chart review of patients treated for mucocele in the Department of Otolaryngology, between 2002 and 2013. Clinical and demographic characteristics, diagnostic methods, treatment and recurrence are described.

Results: 45 patients with average follow-up of 24.93 months were analyzed. $51.11 \%$ were male. The mean age was 49.68 years. $46.6 \%$ were fronto-ethmoidal mucoceles ( 6 in frontal sinus, 6 in ethmoidal sinus and 9 fronto-ethmoidal), the rest in the maxillary sinus, with a latency period of 7.33 months. Most frequent symptoms were ophtalmologic (55.5\%) as proptosis and diplopia, while $51.1 \%$ of patients had a history of chronic rhinosinusitis. Treatment was by endoscopic resection in $66.22 \%, 31.1 \%$ by open surgery and $6.66 \%$ in combined approach. There were 8 recurrent cases, accounting for $17.7 \%$ of the series.

Discussion: Our series was similar to other published earlier. In recent years, endoscopic approach is preferred, however, combined external approach or keys remain in handling large mucoceles or to treat recurrences. The late onset of recurrences potentially requires a long follow-up time for these patients.

Conclusions: Mucoceles represent a diagnostic and therapeutic challenge and endoscopic management seems a safe alternative in the management of orbital involvement or cranial extension mucoceles.

Key words: Mucocele, paranasal sinuses, endoscopic sinus surgery.

\section{INTRODUCCIÓN}

Los mucoceles son pseudotumores expansivos ubicados en las cavidades paranasales ${ }^{1}$. La localización más común de los mucoceles es el seno frontal y frontoetmoidal, sin embargo, también pueden encontrarse en seno maxilar y esfenoidal ${ }^{2}$. Éstos, causan expansión y dilatación lenta y progresiva de las cavidades paranasales, produciendo síntomas nasosinusales y oftalmológicos, tales como: cefalea, obstrucción nasal, dolor facial, diplopía, entre otros ${ }^{3}$.

Se han descrito numerosos factores predisponentes al desarrollo de los mucoceles, que tienen en común una distorsión de la anatomía de la vía de drenaje de los senos paranasales, ya sea de causa traumática, inflamatoria 0 tumoral ${ }^{4}$.

El tratamiento de los mucoceles consistió clásicamente en la extracción mediante abordajes externos, siendo desplazado últimamente por el manejo endoscópico como tratamiento de elección ${ }^{5}$. En nuestro medio existen pocos reportes de la experiencia en el manejo de mucoceles, destacando las experiencias de Celedón y cols ${ }^{6}$, donde el abordaje externo fue el más usado y el trabajo de Nazar y cols ${ }^{7}$, presentando la experiencia en manejo endoscópico.

\section{OBJETIVO}

Por todo esto, nuestro objetivo es evaluar la experiencia en nuestro servicio sobre el tratamiento de los mucoceles.

\section{MATERIAL Y MÉTODO}

Revisión retrospectiva de los pacientes tratados por mucoceles de cavidades paranasales en el Servicio de Otorrinolaringología de nuestro centro, entre 2002 y 2013. Se describen características clínicodemográficas, tratamiento y aparición de recidivas. La tabulación y análisis de los datos obtenidos se realiza con el software Stata 12 (StataCorp. 2011. CollegeStation, TX: StataCorp LP). Se obtuvo promedio y desviación estándar para las variables cuantitativas y porcentajes para las cualitativas. Las variables cuantitativas se compararon a través 
del test de Student y test exacto de Fisher para las cualitativas. Se realizó análisis de sobrevida mediante el método semiparamétrico de KaplanMeier para evaluación de recidivas, utilizando el test de log-rank para comparar curvas de tiempo de recidiva. Un valor de $\mathrm{p}$ menor a 0,05 se consideró como estadísticamente significativo.

\section{RESULTADOS}

Se analizaron 45 pacientes con seguimiento promedio de 24.93 meses. El $51,11 \%(n=23)$ correspondió al sexo masculino y $48,89 \%(n=22)$ al sexo femenino. La edad promedio fue 49,68 años en un rango de 10 a 92 años.

El $46,6 \%$ fueron mucoceles fronto-etmoidales (6 frontales, 6 etmoidales y 9 fronto-etmoidales), el resto en seno maxilar (24 casos), con un periodo de latencia promedio de 7,33 meses y un seguimiento promedio de $24,93 \pm 22,1$ meses (Figura 1).

Los síntomas más frecuentes fueron oftalmológicos (55,5\%): exoftalmo, diplopía y dolor ocular (Tabla 1). La lateralidad fue como sigue: lado derecho $68,89 \%$ ( $n=31$ ) y lado izquierdo $31,11 \%$ (n $=14$ ). Dentro de la comorbilidad asociada destaca que el $51,1 \%$ tenía antecedentes de rinosinusitis crónica (con pólipos y sin ellos) (Tabla 2).

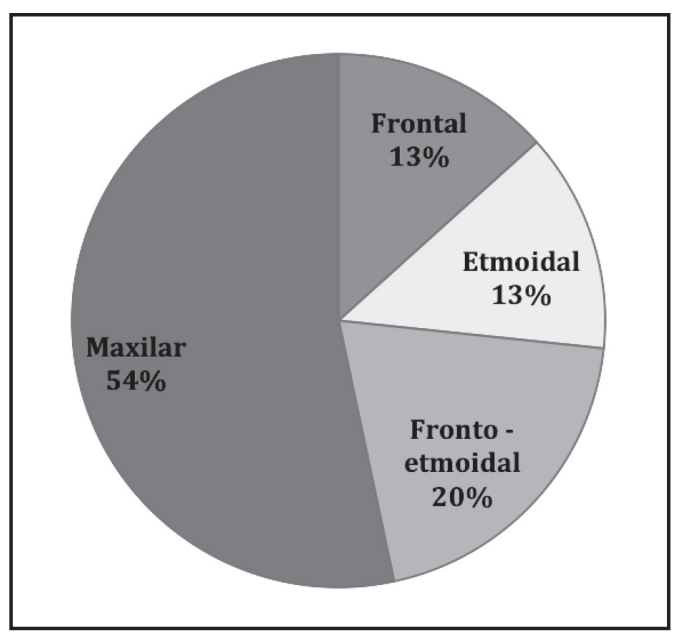

Figura 1. Distribución según ubicación de los mucoceles de cavidades paranasales.
La totalidad de los pacientes tuvo tomografía computarizada de cavidades paranasales como método diagnóstico. Éstas, mostraron en general la imagen pseudotumoraliso o hipodensa que desplaza estructuras adyacentes como la pared orbitaria o simplemente velamiento de cavidades. Además, el 15,5\% ( $n=7)$ pacientes tuvieron un resonancia magnética, principalmente en casos con sospecha de mucoceles extensos o para diagnóstico diferencial en casos en que la tomografía computarizada no fue categórica.

El tratamiento fue realizado por vía endoscópica en el $66,22 \%$ ( $n=28)$ y por vía abierta en el $31,1 \%(n=14)$, de los cuales 6 se realizaron según técnica de Caldwell-Luc, 4 por técnica osteoplástica frontal, 2 por técnica de Lynch, 1 por técnica de Kilian y 1 caso por cranealización del seno frontal. Además, se tuvo un tratamiento combinado en el $6,66 \%(n=3)$, todos ellos combinando la técnica endoscópica y Caldwell-Luc. En la Tabla 3 se comparan los resultados quirúrgicos según vía de abordaje a los mucoceles de cavidades paranasales, destacando en ella la diferencia significativa en los parámetros inherentes al procedimiento quirúrgico mismo (tiempo quirúrgico y estadía hospitalaria) y no así para la comparación de las complicaciones y recidivas.

Hubo 8 casos recidivados correspondiente al $17,78 \%$. De ellos, 5 casos fueron intervenidos en primera instancia por vía endoscópica y 3 por

\section{Tabla 1. Resumen de la presentación clínica de los mucoceles de cavidades paranasales}

\begin{tabular}{|l|c|c|}
\hline Signo o síntoma & $\mathrm{N}^{0}$ & $\begin{array}{c}\% \text { del total } \\
\text { de pacientes }\end{array}$ \\
\hline Dolor facial & 17 & 37,7 \\
\hline Exoftalmo & 17 & 37,7 \\
\hline $\begin{array}{l}\text { Aumento volumen } \\
\text { facial }\end{array}$ & 5 & 11,1 \\
\hline Diplopía & 5 & 11,1 \\
\hline Obstrucción nasal & 8 & 17,7 \\
\hline Dolor ocular & 3 & 6,66 \\
\hline Hipoestesia facial & 6 & 13,3 \\
\hline Cefalea & 3 & 6,66 \\
\hline $\begin{array}{l}\text { Rinosinusitis a } \\
\text { repetición }\end{array}$ & 1 & 2,22 \\
\hline
\end{tabular}


Tabla 2. Comorbilidad asociada a mucoceles de cavidades paranasales

\begin{tabular}{|l|c|c|}
\hline Patología & $\mathrm{N}^{0}$ & \% del total de pacientes \\
\hline Rinosinusitis crónica & 18 & 40 \\
\hline Poliposis nasosinusal & 5 & 11,1 \\
\hline Insuficiencia renal crónica & 5 & 11,1 \\
\hline Displasia osteofibrosa & 1 & 2,22 \\
\hline Trauma maxilofacial severo & 1 & 2,22 \\
\hline
\end{tabular}

Tabla 3. Resultados quirúrgicos según vía de abordaje en el manejo de los mucoceles de cavidades paranasales

\begin{tabular}{|l|c|c|c|}
\hline Variable & Vía abierta & Vía endoscópica & Valor de $p$ \\
\hline Tiempo quirúrgico, min y DS & $182,05(69,30)$ & $130,71(31,84)$ & 0,0015 \\
\hline Estadía hospitalaria, días y DS & $7,41(5,85)$ & $2,96(1,59)$ & 0,0002 \\
\hline$N^{\circ}$ Complicaciones/Secuelas & $2(66,6 \%)$ & $1(33,3 \%)$ & 0,2241 \\
\hline$N^{\circ}$ Recidivas & $3(37.5 \%)$ & $5(62.5 \%)$ & 0,1441 \\
\hline
\end{tabular}

vía abierta. Seis de estos casos correspondieron a tumores relativamente grandes en ubicación fronto-etmoidal y los restantes 2 se encontraron en ubicación maxilar. El tiempo de latencia para las recidivas fue en general de $15,75 \pm 10,01$ meses, en rango de 4 a 36 meses. Aquellos casos intervenidos por vía abierta recidivaron en promedio a los $15,66 \pm 7,23$ meses en rango de 11 a 24 , mientras los intervenidos por vía endoscópica recidivaron en promedio a los 15,8 $\pm 12,2$ meses en rango de 4 a 36 meses.

En la Figura 2, se presenta la estimación de recidivas mediante el método de Kaplan-Meier, según tipo de cirugía. No hubo diferencias estadísticamente significativas en el tiempo de recidiva entre los 2 métodos quirúrgicos ( $p=0,4547$ ). El

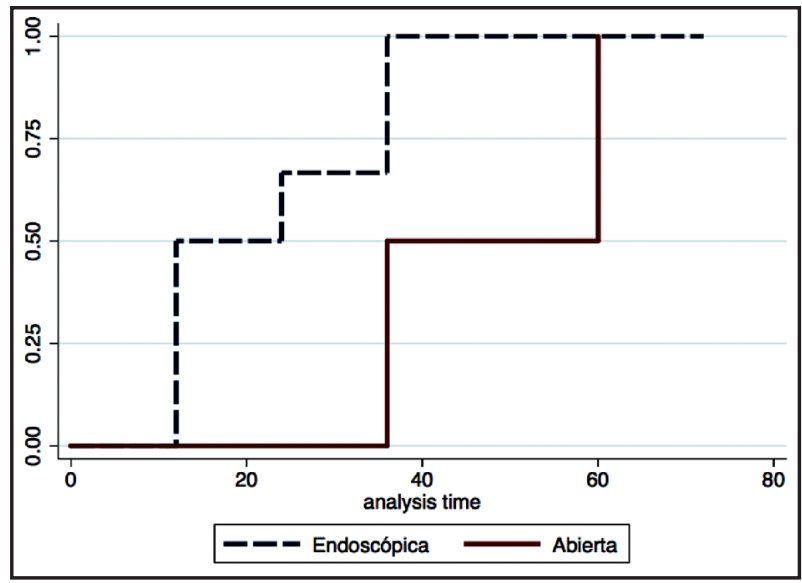

Figura 2. Curva de Kaplan-Meier para la estimación del tiempo de recidiva, según tipo de tratamiento. 
manejo de las recidivas fue realizado mediante técnicas quirúrgicas abiertas: $75 \%(n=6)$ osteoplástica frontal y $25 \%(n=2)$ a través de CaldwellLuc. Dentro de las complicaciones se tuvo un caso de fístula de líquido cefalorraquídeo y uno de estrabismo en cirugías abiertas y un caso de obstrucción por estenosis de vía lagrimal en cirugía endoscópica.

\section{DISCUSIÓN}

Los mucoceles son masas benignas, cuyo crecimiento lento produce erosión de las estructuras óseas adyacentes, lo que determina su sintomatología'. Se han identificado varios factores predisponentes para el desarrollo de mucoceles paranasales, que corresponden a circunstancias que producen una distorsión traumática, inflamatoria o tumoral de las vías de drenaje sinusal ${ }^{3}$. Estos elementos deben ser investigados por el otorrinolaringólogo.

Nuestra casuística resultó semejante a otras series publicadas, en términos de la presentación clínica y aparición de recidivas ${ }^{8,9}$. La presencia de rinosinusitis crónica con y sin pólipos estuvo frecuentemente asociada a la aparición de mucoceles, tal como han comunicado otros autores ${ }^{3,10}$. En nuestra serie no fue posible recabar el antecendente de cirugía endoscópica de cavidades paranasales, quizás por subregistro, como se ha reportado en algunas series ${ }^{5}$.

La evaluación con tomografía computarizada y/o con resonancia magnética de cavidades paranasales es clave para determinar la ubicación y tamaño de los mucoceles, la identificación de anomalías anatómicas que sean relevantes a la hora de considerar la técnica quirúrgica a utilizar en el tratamiento y para el diagnóstico diferencial que incluye entidades tales como la bola fúngica, quistes de retención y lesiones malignas de cavidades paranasales ${ }^{10}$. Los mucoceles en tomografía computarizada se aprecian como lesiones óseas expansivas circundadas por un hueso adelgazado y remodelado. Con medio de contraste estas lesiones no presentan realce en su imagen. En la resonancia magnética, pueden aparecer como lesiones hiperintensas en $\mathrm{T} 2 \mathrm{e}$ iso 0 hipointensas en $\mathrm{T} 1$ de acuerdo con la cantidad de líquido o proteínas que contengan ${ }^{11}$. El beneficio de la tomografía compu- tarizada por sobre la resonancia magnética es la posibilidad de observar con más detalle el hueso, lo que es útil en la evaluación preoperatoria ${ }^{16}$.

En los últimos años hemos preferido el abordaje endoscópico ya que esta técnica permite conservar el marco óseo del seno, implica un menor tiempo quirúrgico y costos de hospitalización, además de evitar la incisión externa, beneficios ampliamente reportados en la literatura ${ }^{12,13}$. Sin embargo lo anterior, el abordaje externo o combinado sigue siendo clave en el manejo de mucoceles extensos o que comprometan el seno frontal lateral o pared anterior del seno maxilar y para el manejo de las recidivas ${ }^{14}$, lo que se ve refrendado en nuestra serie.

Aparentemente, los casos intervenidos endoscópicamente tienden a recidivar antes en el tiempo, pero no en mayor cuantía que los casos operados por vía abierta, sin embargo, se requieren de estudios más extensos para establecer fehacientemente este patrón de comportamiento. Según Devars du Mayne y $\operatorname{cols}^{15}$, se requeriría mucho más que el tiempo general de seguimiento en los trabajos reportados (en torno a 4 años), quizás 70 más años, pues muchas de las recidivas pueden mantenerse asintomáticas mucho tiempo, por lo que el seguimiento clínico y radiológico es fundamental.

En suma, el tiempo de latencia de síntomas junto con la aparición potencialmente tardía de recidivas, enfatiza la investigación anamnéstica acuciosa y la necesidad de un tiempo de seguimiento largo para estos pacientes. El manejo endoscópico parece una alternativa segura en el manejo de mucoceles que no comprometan órbita o tengan extensión craneal.

\section{BIBLIOGRAFÍA}

1. LeE KJ, ed. Essential Otolaryngology, Head and Neck Surgery. $9^{\text {th }}$ ed. Mc Graw Hill, 2008.

2. Capra GG, Carbone PN, Muluin DP. Paranasal sinus mucocele. Head Neck Pathol 2012; 6(3): 369-72.

3. Kım YS, Kım K, Lee JG, Yoon JH, Kım CH. Paranasal sinus mucoceles with ophthalmologic manifestations: a 17-year review of 96 cases. Am J Rhinol Allergy 2011; 25(4): 272-5.

4. Obeso S, Llorente Jl, Pablo Rodrigo J, Sánchez R, Mancebo G, Suárez C. Paranasal sinuses 
mucoceles. Our experience in 72 patients. Acta Otorrinolaringol Esp 2009; 60(5): 332-9.

5. Dhepnorrarat RC, Subramaniam S, Sethi DS. Endoscopic surgery forfronto-ethmoidal mucoceles: a 15-year experience. Otolaryngol Head Neck Surg 2012; 147(2): 345-50.

6. Celedón C, Inzunza F, Olavarría C, Silva C, Tamblay $\mathrm{N}$, TAPIA L. Estudio y manejo de mucoceles de cavidades perinasales. Rev Otorrinolaringol Cir Cabeza Cuello 2003; 63: 29-34.

7. Nazar R, Naser A, Pardo J, Fulla J, RodríguezJoRGE J, DÉlAno PH. Endoscopic management of paranasal sinus mucoceles: experience with 46 patients. Acta Otorrinolaringol Esp 2011; 62(5): 363-6.

8. Morita S, Mizoguchi K, IIZUKa K. Paranasal sinus mucoceles with visual disturbance. Auris Nasus Larynx 2010; 37(6): 708-12.

9. Woodworth BA, Harvey RJ, Neal JG, Palmer JN, SCHLOSSER RJ. Endoscopic management of frontal sinus mucoeceles with anterior table erosion. Rhinology 2008; 46(3): 231-7.

10. Khalil SH, Adams MW. Clinico-radiological considerations in the treatment of frontal sinus mucoceles. Rev Laryngol Otol Rhinol (Bord) 2009; 130(4-5): 267-71.

11. Pierse JE, Stern A. Benigncysts and tumors of the paranasal sinuses. Oral Maxillofac Surg Clin North Am 2012; 24(2): 249-64, ix.

12. HAR-EL G. Endoscopic management of 108 sinus mucoceles. The Laryngoscope 2001; 111: 21314.

13. Busaba NY, Salman SD. Maxillary sinus mucoceles: clinical presentation and long-term results of endoscopic surgical treatment. The Laryngoscope 1999; 109: 1446-9.

14. Lee TJ, Li SP, Fu CH, Huang CC, Chang PH, Chen YW, Chen CW. Extensive paranasal sinus mucoceles: a 15-year review of 82 cases. Am J Otolaryngol 2009; 30(4): 234-8.

15. Devars du Mayne M, Moya-Plana A, Malinvaud D, LaccourReye 0, BonfiLs P. Sinus mucocele: natural history and long-term recurrence rate. Eur Ann Otorhinolaryngol Head Neck Dis 2012; 129(3): 125-30.

16. Lloyd G, Lund VJ, Savy L, Howard D. Optimum imaging for mucoceles. J Laryngol Otol 2000; 114: 233-6. 